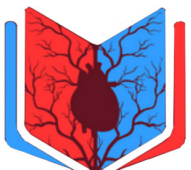

Published By : IVAA

the Indonesian Vascular Access Association

\section{Central venous catheters retention in a patient with mitral valve replacement: a case report}

\author{
Ngurah Dwiky Abadi Resta', I Nyoman Semadi' ${ }^{1}$ I Komang Adhi Parama Harta', \\ I Wayan Sudarma ${ }^{1}$, Ketut Putu Yasa ${ }^{1 *}$
}

'Division of Cardiac Thoracic and Vascular Surgery, Department of Surgery Udayana University / Sanglah General Hospital, Denpasar, Bali, Indonesia
*Corresponding to:

Ketut Putu Yasa;

ketut.putuyasa07@gmail.com

\section{ABSTRACT}

Background: Retention of central venous catheters (CVC) is one complication that may occur when open-heart surgery is performed (such as mitral valve replacement). In this case report, we describe case retention of CVC in a patient with Mitral Valve Replacement (MVR) related to sutured of Superior Vena Cava (SVC) wall on cannulation site.

Case Presentation: A 15-year-old boy was admitted to Sanglah Hospital with a history of Heart failure with severe regurgitation of the mitral valve, severe tricuspid regurgitation, and left ventricular dysfunction due to rheumatic heart disease. Mitral valve replacement, tricuspid valve repair and left atrial reduction was performed. After five days of postoperative observation, the CVC could be removed. However, there is resistance when removing the catheter. After diagnostic examination, it was found that the CVC was sutured to the superior vena cava wall. The patient was then scheduled for a redo sternotomy to evacuate the CVC. The patient was discharged seven days after redo sternotomy was performed without any further postoperative complications.

Conclusions: Retention of CVC during open-heart surgery is one complication that increases the risk for morbidity or mortality to the patient after heart surgery.

Keywords: Central venous catheters, Retention, Valve Surgery.

Cite This Article: Resta.N.D.A., Semadi, I.N., Harta, I.K.A.P., Sudarma, I.W., Yasa, K.P. 2021. Central venous catheters retention in a patient with mitral valve replacement: a case report. Journal of Indonesia Vascular Access 1(1): 16-18. D0I : 10.51559/ jinava.v1i1.11

\section{BACKGROUND}

The Central Venous Catheters (CVC) are vascular access inserted into the subclavian vein, internal jugular vein or femoral vein. Central Venous Catheters is an important component of fluids access, intravenous drugs, and monitoring of venous pressure. Several types of CVC are often used, such as non-tunneled CVC, tunneled CVC, Peripheral Inserted Central Catheters (PICC), and Implantable ports. Complications from this CVC insertion procedure can include mechanical (hematoma, arterial puncture, pneumothorax, hemothorax, catheter misplacement, stenosis), infection (insertion site infection, CVC colonization, and bloodstream infection) and thrombotic (deep vein thrombosis). ${ }^{1,2}$
The sutured catheter into the heart or vessel wall during Mitral Valve Replacement (MVR) is a rare complication and difficult to repair. If there is any mistake when removing the catheter, it can tear the blood vessel, cardiac tamponade and even death. ${ }^{1}$ We report a case of CVC sutured to the wall of the Superior Vena Cava (SVC) during MVR.

\section{CASE PRESENTATION}

A 15-year-old Boy $(155 \mathrm{~cm}, 33 \mathrm{~kg})$ was admitted with a chief complaint of palpitation. He said palpitate, and shortness of breath gets worse when supine position. The patient has been diagnosed with Rheumatic Heart Disease (RHD) since December 2018. Family history of cardiac diseases is denied. This patient was routinely treated with Benzylpenicillin G (BPG), oral $40 \mathrm{mg}$ furosemide once a day, $12.5 \mathrm{mg}$ captopril three times a day, and $12.5 \mathrm{mg}$ spironolactone twice a day. The immunization history of the patient is complete. When the patient comes to the hospital, the physical examination of the patient shows abnormality with pulse rate 138x / minute, regular, respiratory rate $24 \mathrm{x} /$ minute, oxygen saturation $98-99 \%$. On physical examination, the heart found a grade 4/6 ICS IV systolic murmur at the apex radiating to the axilla. Then, the patient performed echocardiography. The result is severe mitral regurgitation et causa restricted posterior mitral leaflet (Figure 1), severe tricuspid regurgitation, normal left ventricular systolic function, decreased right ventricular systolic function, grade II of decreased left ventricular diastolic 

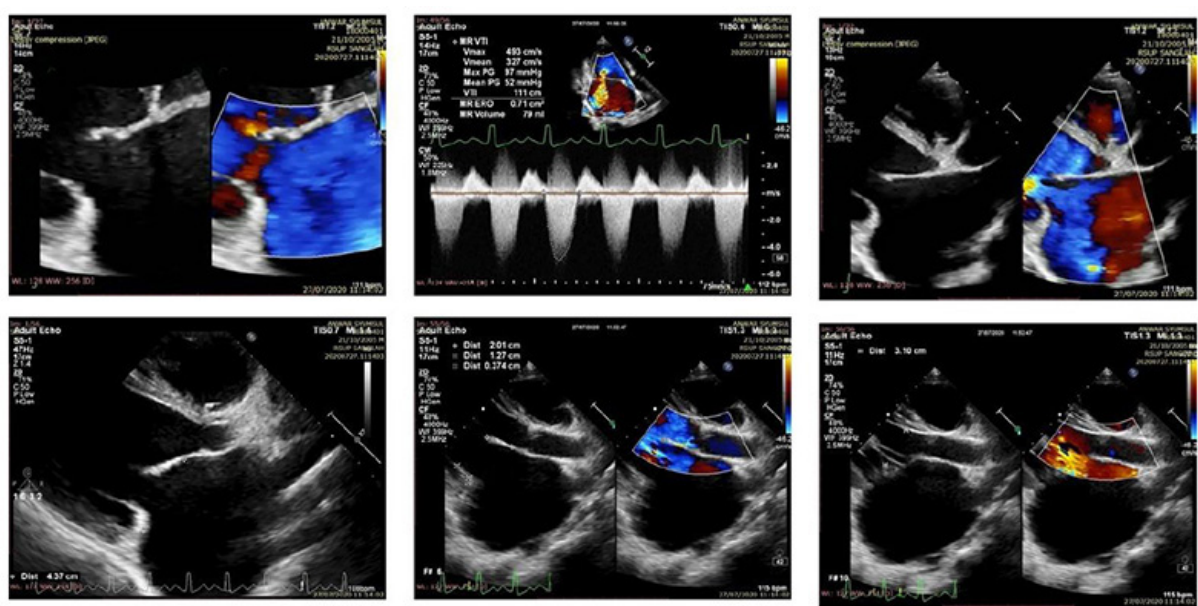

Figure 1. Echocardiography of Patient MR severe ec restricted posterior mitral leaflet (mitral annulus $4.37 \mathrm{~cm}$, C-sept $3.01 \mathrm{~cm}$, anterior mitral leaflet $2.01 \mathrm{~cm}$, posterior mitral leaflet $1.64 \mathrm{~cm}$ )

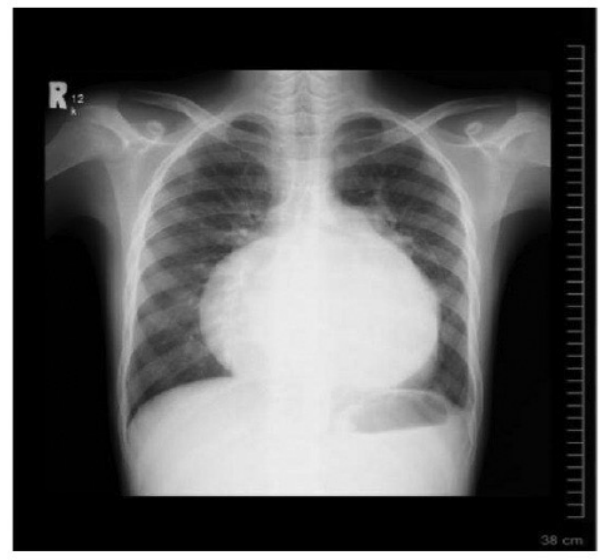

(a)

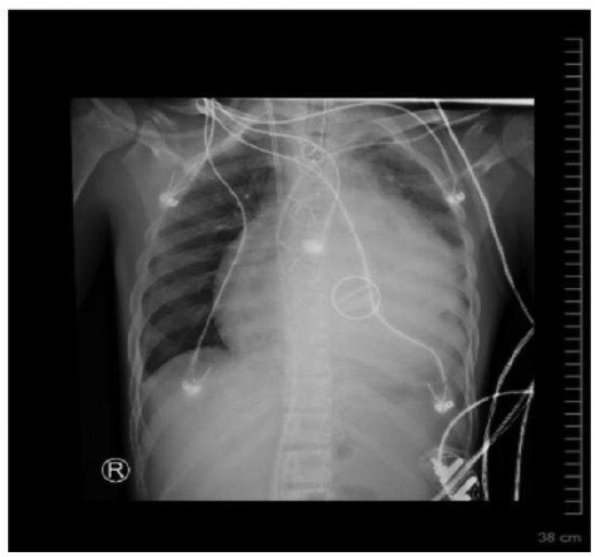

(b)

Figure 3. Postoperative control Anteroposterior chest radiographs

(a) X-rays before surgery;

(b) X-rays after MVR, TVr, LA reduction procedure

function. The patient was diagnosed with chronic heart failure functional class III, severe mitral regurgitation et causa RHD, severe tricuspid regurgitation, left ventricle dysfunction, giant left atrium. After the team meeting, the patient planned to take MVR, Tricuspid Valve Repair (TVR) and Left Atrium (LA) reduction procedure. During the preparation of the operation, the therapy is given fluids as needed, oral spironolactone $12.5 \mathrm{mg}$ every 12 hours, oral captopril $12.5 \mathrm{mg}$ every 8 hours, oral furosemide $20 \mathrm{mg}$ every 24 hours BPG 1.2 million units every 21 days intramuscular.

The patient was performed for MVR surgery with a $33 \mathrm{~mm}$ mechanical valve (15 pledget sutures) and tricuspid valve repair with Kay's procedure. Postoperative control anteroposterior chest radiographs were obtained before admission to the intensive room (Figure 3 a-b). Five days later, the patient was stable and planned to remove the catheter that was attached. During the removal process, there was resistance. Then an ultrasound examination was performed, it was seen that the end of the CVC was attached to the SVC wall. When the CVC was pulled, there was also a pull on the SVC wall, so that it was suspected that the end of the CVC was sewn into the cannulation site of the SVC wall. After conducting a team meeting, it was decided to undergo surgery to remove the retained CVC. Redo sternotomy was performed on the patient by opening sutures and wire sternum.

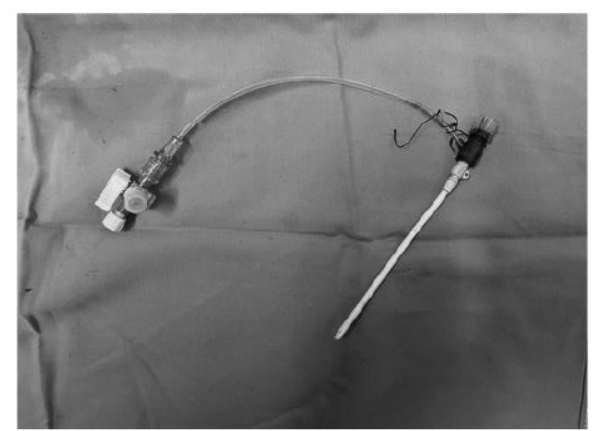

Figure 2. $\quad$ CVC after evacuated. Arrow : tip of CVC stitches to the SVC wall

After that adhesiolysis with warm water, identified the position of the CVC sutured in the SCV cannulation suture line. CVC was evacuated, and a repair SVC wall was performed. There is a stitch mark on the end of the CVC that has been evacuated (Figure 2). After the procedure, the patient was observed in the pediatric intensive care unit. Seven days later, the patient was discharged in good condition.

\section{DISCUSSION}

MVR can be performed in patients with indications of severe symptomatic mitral valve diseases. There needs to be a more strict evaluation before performing mitral valve surgery in patients with comorbid diseases such as chronic respiratory obstruction, cerebrovascular disease, and kidney disease. In some patients, the longterm prognosis is more affected by their comorbidities than a valvular disease. ${ }^{3}$

There are two options for the MVR procedure, which can be performed with bioprosthesis or mechanical valves. Mechanical valves offer better durability, but long-term anticoagulation therapy for preventing thromboembolism is associated with an increased risk of bleeding. ${ }^{4}$ To determine which valve to use is seen from several factors, including the most important ones are the patient preference, age and life expectancy. In addition, the patient who has comorbidities such as kidney disease, contraindications to anticoagulants, pregnancy, previous infection, risk of re-operation are also important factors. Mechanical valves are recommended for patients less than sixtyfive years of age, while bioprosthesis is for patients older than seventy years. ${ }^{3,5}$ 
Giant Left Atrium (GLA) is a rare condition associated with a rheumatic mitral valve. In a review article published by El Maghraby and Hajar, GLA is almost always caused by mitral valve diseases. The goals of the GLA surgery are to repair the abnormal mitral valve, reduce clinical manifestations, prevent thromboembolism, and return heart rhythm from atrial fibrillation to sinus rhythm. There are two strategies to repair the giant left atrium. First, by performing mitral valve replacement with left atrial volume reduction, or the second is mitral valve surgery alone. Surgeons believe that a successful mitral valve surgery alone can reduce the left atrium size and lowers the Mean Arterial Pressure (MAP). ${ }^{6}$

The patient was diagnosed with severe mitral regurgitation, severe tricuspid regurgitation, and giant left atrium as described in echocardiography. Therefore, MVR, TVR, and LA reduction measures are suitable choices. In this patient, mechanical valve replacement was performed because of his young age and high life expectancy. Patients who receive MVR generally have poor heart function before surgery. Therefore, the operative approach to remove the CVC must be handled with care.

Accidental suturing of the CVC to the heart is a rare complication. There are several accidental suturing of the CVC cases that are difficult to remove. Haiying Kong et al., in their case report, also reported almost the same case, an accidental suturing CVC during the MVR operation. ${ }^{1}$ There are no data that describe cases of CVC stitching in the MVR case. The CVC insertion is generally done in the subclavian vein, internal jugular vein and femoral vein. ${ }^{1,2}$ In this case, the position of the CVC tip is placed over the SVC region, so there is a possibility of accidental suturing when performing the cannulation suture. One of the methods to remove the remaining CVC is surgery. ${ }^{1}$ Mahadeva et al. reported a case of a part of the CVC which gets left behind due to a broken catheter tip and taken with distal venotomy. ${ }^{7}$ In this case, removal of the CVC suture is performed by redo sternotomy to reduce the risk of injury to the SVC wall if forced extraction is carried out. This is supported by Abdorasul et al., who found a case of accidental suturing of the CVC during mitral valve replacement surgery and removed the CVC surgically. ${ }^{8}$

\section{CONCLUSION}

CVC placement is an essential step for the hospitalized patient, especially those who will undergo major surgery. There are some common complications of the CVC placement, such as infection, thrombosis, and mechanical disturbances. One of the complications that can also occur is the accidental suturing of CVC to the SVC wall during the MVR procedure. To prevent this complication, care needs to be taken when sewing the cannulation site so that it does not hit the CVC. Retention and forced CVC extraction have the effect of increasing the morbidity and mortality of the patient, such as tearing the SVC wall or the heart after heart surgery. In this case, MVR operation with mechanical valve was carried out according to the procedure. The redo sternotomy is done to evacuate the CVC without causing any more severe complications. The patient was discharged after seven days of postoperative care of redo sternotomy.

\section{CONFLICT OF INTEREST}

There is No Conflict of Interest

\section{AUTHOR CONTRIBUTION}

Concepts : NDAR, INS, IKAPH, IWS,

KPY

Design : NDAR, INS, IKPAH, IWS, KPY

Definition of intellectual content : NDAR, KPY

Literature search : NDAR, KPY

Data acquisition. : NDAR, KPY

Data analysis. : NDAR, KPY

Manuscript preparation : NDAR, KPY

Manuscript editing : NDAR, KPY

Manuscript review : NDAR, KPY

Guarantor : KPY

\section{FUNDING}

None

\section{CONSENT FOR PUBLICATION}

The patient's parents had agreed and signed informed consent regarding publishing the case in an academic journal without exposing his child's identity.

\section{REFERENCE}

1. Kong $\mathrm{H}$, Chen $\mathrm{S}$, Wen $\mathrm{X}$. Suture of the right internal jugular vein catheter in a mitral valve replacement: a case report. J Med Case Rep. 2014;8(1). Available from: http://dx.doi. org/10.1186/1752-1947-8-129

2. Cruz R, Pires R, Rodrigues N, Machado J. Central venous catheterization: An updated review of historical aspects, indications, techniques, and complications. Transl Surg. 2017;2(3):66. Available from: http://dx.doi. org/10.4103/ts.ts_10_17

3. Sayeed R. Mitral valve disease: when should we call in the cardiac surgeon? E-Journal Cardiol Pract. 2018;16:28-31.

4. Cetinkaya A, Poggenpohl J, Bramlage K, Hein S, Doss M, Bramlage $\mathrm{P}$, et al. Long-term outcome after mitral valve replacement using biological versus mechanical valves. J Cardiothorac Surg. 2019;14(1). Available from: http://dx.doi. org/10.1186/s13019-019-0943-6

5. Kumar RK, Antunes MJ, Beaton A, Mirabel M, Nkomo VT, Okello E, et al. Contemporary Diagnosis and Management of Rheumatic Heart Disease: Implications for Closing the Gap: A Scientific Statement From the American Heart Association. Circulation. 2020;142(20). Available from: http://dx.doi.org/10.1161/ cir.0000000000000921

6. El Maghraby A, Hajar R. Giant left atrium: A review. Hear Views. 2012;13(2):46. Available from: $\quad$ http://dx.doi.org/10.4103/1995705x.99227

7. Mahadeva S, Cohen A, Bellamy M. The stuck central venous catheter: beware of potential hazards. Br J Anaesth. 2002;89(4):650-2. Available from: http://dx.doi.org/10.1093/bja/ aef239

8. Anvaripour A, Yazdanian F, Totonchi M-Z, Shahryari H. Inadvertent Entrapment of a Central Venous Catheter by a Purse-String Suture during Cardiopulmonary Bypass: A Case Report. Case Rep Anesthesiol. 2011;2011:1-4. Available from: http://dx.doi. org/10.1155/2011/760426

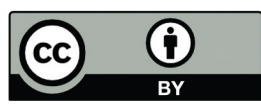

This work is licensed under a Creative Commons Attribution 